Gynäkologe 2019·52:252-253

https://doi.org/10.1007/s00129-019-4414-x

Online publiziert: 21. März 2019

(c) Springer Medizin Verlag GmbH, ein Teil von Springer Nature 2019
Marion Kiechle ${ }^{1}$ Thomas Strowitzki ${ }^{2}$ Wolfgang Küpker ${ }^{3} \cdot$ Ricardo Felberbaum $^{4}$

${ }^{1}$ Universitätsfrauenklinik, Technische Universität München, München, Deutschland

${ }^{2}$ Gynäkologische Endokrinologie und Fertilitätsstörungen, Universitäts-Frauenklinik, Heidelberg, Deutschland

${ }^{3}$ Klinik für Frauenheilkunde, Geburtsmedizin und Reproduktionsmedizin, Baden-Baden, Deutschland

${ }^{4}$ Klinik für Frauenheilkunde und Geburtshilfe, Kliniken Kempten und Immenstadt, Klinikverbund Kempten - Oberallgäu, Kempten, Deutschland

\title{
Myome - benigne Tumoren des Uterus mit großer Bedeutung für die Frauengesundheit
}

Uterusmyome sind die mit Abstand häufigsten gutartigen Tumoren der Frau. Mehr als $70 \%$ aller Frauen entwickeln bis zur Menopause Myome [1]. Mindestens $25 \%$ aller Frauen haben Beschwerden und Symptome und müssen sich einer Behandlung unterziehen. Dies betrifft in erster Linie prämenopausale Frauen, die hauptsächlich an Blutungsstörungen leiden und deren Fertilität in Mitleidenschaft gezogen ist. Die Behandlungsmodalitäten sind vielfältig und mit unterschiedlichen Risiken und Erfolgsaussichten vergesellschaftet. Welche Behandlung im individuellen Fall eingesetzt wird, richtet sich daher nach der Symptomschwere, der Lebenssituation und dem Wunsch der Frau. Für den betreuenden Gynäkologen ist es daher wichtig, alle Behandlungsmodalitäten zu kennen, um die Frau individuell und umfassend zu beraten.

Das Schwerpunktthema dieser Ausgabe von Der Gynäkologe widmet sich daher in erster Linie der Therapie des Uterus myomatosus, auch unter dem Gesichtspunkt der Subfertilität. Die operative Entfernung der Gebärmutter oder der Myome mittels minimal-invasiver Techniken ist keinesfalls mehr das einzige ablative Verfahren. Moderne und verfeinerte Techniken der Bildgebung in Kombination mit fokussiertem Ultraschall, Embolisation oder Radiofrequenzverfahren haben Einzug genommen in die Behandlung des symptomatischen Uterus myomatosus.
Auch in der medikamentösen Therapie der Uterusmyome in den letzten Jahren sind entscheidende Fortschritte erzielt worden. Seit 2012 steht neben GnRH(„gonadotropin releasing hormone")-Analoga auch der selektive Progesteronrezeptormodulator (SPRM) Ulipristalacetat (UPA) zur Verfügung. Ein weiterer SPRM zeigt erste vielversprechende Wirkungen in einer Phase2-Studie. Und oral verfügbare $\mathrm{GnRH}$ Antagonisten werden in Zukunft das Spektrum der Möglichkeiten einer medikamentösen Therapie noch erweitern.

\section{\) Die Vielfalt der Therapieop- tionen erlaubt eine individuelle, risikoadaptierte Behandlung}

Obwohl die Lebensqualität vieler Frauen durch Uterusmyome beeinträchtigt ist, sie dadurch eine große Bedeutung für die Frauengesundheit haben und ihre Behandlung nicht unerhebliche Kosten für das Gesundheitssystem bedingen [2], existiert zur Behandlung des Uterus myomatosus bislang keine S3-Leitlinie der deutschsprachigen Fachgesellschaften.

Auch wissen wir über die Ursachen dieser Erkrankung sehr wenig. In epidemiologischen Studien werden zahlreiche Risikofaktoren genannt. $\mathrm{Zu}$ den beeinflussbaren Risikoparametern zählen Lebensstilfaktoren, wie Übergewicht, Rauchen und Alkoholkonsum, aber auch endokrine Faktoren, wie Pa- 
rität oder Antibabypilleneinnahme. $\mathrm{Zu}$ den nicht beeinflussbaren Parametern zählen beispielsweise eine afrikanische Abstammung, das Alter und auch eine positive Familienanamnese [3]. Liegt es also auch in den Genen? Dass Myome unterschiedliche genetische Veränderungen aufzeigen, schildert der Beitrag von Bullerdiek und Küpker in diesem Schwerpunktheft. Die molekulargenetische Erforschung der Myome ist ein vielversprechender Ansatz, die Pathophysiologie dieser Erkrankung weiter zu charakterisieren und dadurch neue Möglichkeiten für Prävention und Therapie zu entwickeln.

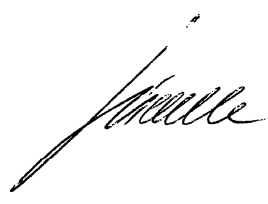

Marion Kiechle

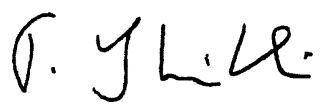

Thomas Strowitzki

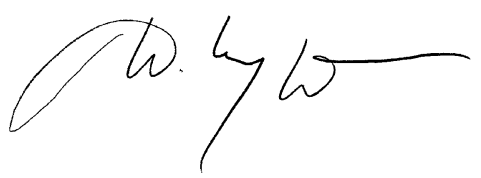

Wolfgang Küpker

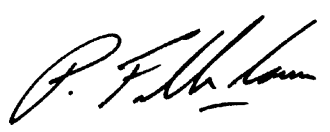

Ricardo Felberbaum

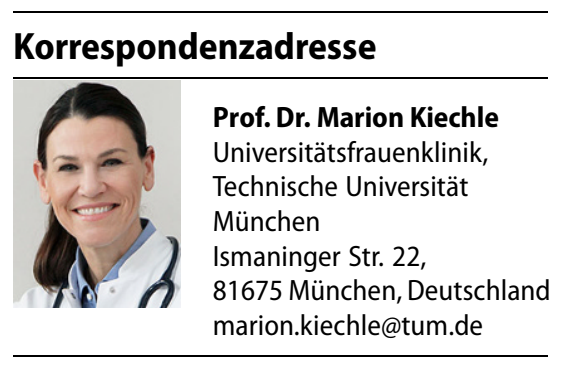

Interessenkonflikt. M. Kiechle, T. Strowitzki, W. Küpker und R. Felberbaum geben an, dass kein Interessenkonflikt besteht.

\section{Literatur}

1. Stewart EA, Cookson CL, Gandolfo RA, SchulzeRath R (2017) Epidemiology of uterine fibroids: a systematic review. BJOG 124(10):1501-1512. https://doi.org/10.1111/1471-0528.14640

2. Al-Hendy A, Myers ER, Stewart E (2017) Uterine fibroids: burden and unmet medical need. Semin Reprod Med 35(6):473-480. https://doi.org/10. 1055/s-0037-1607264

3. Pavone D, Clemenza S, Sorbi F, Fambrini M, Petraglia F (2018) Epidemiology and risk factors of uterine fibroids. Best Pract Res Clin Obstet Gynaecol 46:3-11. https://doi.org/10.1016/j. bpobgyn.2017.09.004
Bessere Heilungschancen für Patientinnen mit Brustkrebs durch Taktilographie

Der hochsensible Tastsinn blinder Frauen rettet Leben.

Hochgradig sehbehinderte MedizinischTaktile Untersucherinnen (MTUs) arbeiten nach einer 9-monatigen Qualifikation mit Abschlussprüfung nach einem standardisierten und qualitätsgesicherten Konzept als Tastspezialistinnen in der Brustkrebsfrüherkennung.

Die Taktile Brustuntersuchung findet in persönlicher Atmosphäre statt und bietet Raum für eine individuelle Beratung zur Brustgesundheit. Während der je nach Brustgröße 30- bis 60-minütigen Untersuchung tastet die MTU mithilfe von selbstklebenden Orientierungsstreifen die Brust sowie die Lymphknoten Millimeter für Millimeter systematisch in drei Tiefenschichten schonend ab. Frauenärzte selbst haben bei der jährlichen Vorsorge nur wenige Minuten Zeit zum Brustabtasten.

Die MTU kann keinen „Krebs tasten“, sondern lediglich Gewebeveränderungen lokalisieren. Im Taktilogramm dokumentiert sie ihren Befund graphisch für den Facharzt. Dieser bewertet den Befund, stellt die abschließende Diagnose und trägt die Verantwortung dafür. Auffällige Befunde klärt er per Ultraschall ab und leitet ggf. die weitere Diagnostik gemäß S3-Leitlinie ein.

Eine prospektive klinische MonocenterStudie des Universitäts-Brustzentrums der Universität Erlangen mit 339 Patientinnen hat die Wirksamkeit der Taktilographie bestätigt. Immer mehr Krankenkassen übernehmen die Untersuchungskosten.

\section{Fazit der Studie}

Der Einsatz von MTUs kann die Sterblichkeit senken, im Gesundheitssystem Kosten mindern und Frauen motivieren, Präventionsangebote wahrzunehmen was wiederum Behandlungs- und Folgekosten senkt. Die hohe Zufriedenheit der Patientinnen mit der Taktilographie spricht ebenfalls sehr dafür.

Quelle: discovering hands, www.discovering-hands.de 